\section{UK research}

\section{MRC units close}

A SMALL decision by the Medical Research Council (MRC) at the end of March is being seen as a larger blow to the standing of British research in an area where it cannot afford to decline.

The decision is the closure of the MRC Mammalian Genome Unit in Edinburgh, following a year-long search to find a director to replace Ed Southern, who left to take up the chair of biochemistry at the University of Oxford last October. In line with its longstanding policy, MRC first assessed the desirability of maintaining the unit. The decision was strongly in favour of keeping it going but MRC failed to attract a new director who satisfied the criteria for the job.

Commenting on the failure this week, Sir James Gowans, MRC's secretary, said that the council had searched hard for a new director of sufficient calibre and that he had no idea why the search had failed. Perhaps the best people can command greater rewards than a small MRC unit can offer, he speculated. In any case, MRC would certainly continue to support the two key researchers in the unit.

The closure of the unit is being seen in some circles as a further worrying sign of the decline in standing of British research. If nobody can be found to fill such a post, it is argued, either Britain is not producing scientists of the necessary calibre or there are insufficient attractions to keep the best scientists in the country. Professor Southern, who has given his name to the key molecular biological technique of Southern blotting, said last week that whatever the reason for the failure to replace him, he was very worried about its impact on young scientists thinking of pursuing a career in Britain at a time when there is already difficulty in finding good postdoctoral researchers.

A second MRC unit that is to be closed apparently for lack of a suitable director is the Mineral Metabolism Unit in Leeds. Professor B.E.C. Nordin retired as director of this unit in 1981 and Dr Munro Peacock has been holding the fort during the search for a permanent successor. After a very long and extensive search it has been the unanimous view of a committee that included international experts that no suitable director could be found to take over, said Sir James. There is, however, a very strong need to maintain some of the research which concentrates on calcium metabolism.

Two other MRC establishments that seem destined to close are the Unit on Neural Mechanisms of Behaviour and the Developmental Neurobiology Unit. It seems that MRC does not intend to maintain these units after the forthcoming retirement of the directors. Peter Newmark

\title{
It's raining pesticides in Hokkaido
}

Tokyo

JAPAN may be the first country to be suffering from "pesticide rain". There is mounting evidence that pollutants found in Lake Mashu, in the northern island of Hokkaido, have come all the way from the Chinese mainland before being washed out of the sky.

Lake Mashu is a 7,000-year-old lake created when the summit of a volcano collapsed, forming a caldera which filled with water. High cliffs rim the lake and prevent any streams from flowing directly into it: all the lake's water comes from rain or from water draining through the rock. Human influence has been kept to a minimum and visitors are not allowed down to the edge of the lake itself. The result is a lake of exceptional clarity - it is often claimed to be the clearest in the world. In 1979 it was possible to see down to a depth of 117 feet $(35.8 \mathrm{~m})$.

The Environment Agency has been analysing the water of Lake Mashu periodically for the past four years and has discovered that it contains an increasing quantity of the pesticide benzene hexachloride (BHC). The pesticide, originally developed by ICI in the United Kingdom and marketed as "Gammexane", has not been used in Japan for fifteen years because of suspicions that it was carcinogenic. It is still widely used in Europe and the United States, as well as in mainland China and Korea. Concentrations of BHC in the lake now average 32 nanograms per litre; still low, but in the summer the concentration in water at the surface becomes many hundreds of times higher. The rain falling at that time contains as much as $\mathbf{1 2 8}$ nanograms per litre, more than twice the levels detected 15 years ago when BHC was still in use in Japan. The only plausible source for the pesticide is China and Korea where $\mathrm{BHC}$ is often used as an agricultural spray: that means BHC has been transported more than 1,500 kilometres across the Sea of Japan before falling on Hokkaido.

BHC levels in the lake will probably continue to rise for the lake has no outlets and the pesticide is virtually nonbiodegradable. It is still a long way from approaching harmful levels but that has not stopped there being some hard feelings about the way other nations can pollute one of Japan's most protected lakes.

Alun Anderson

\section{Telescope bites cosmic dust}

\section{Washington}

BARRING an eleventh-hour reprieve, the Clark Lake Radio Observatory (CLRO) in Borrego Springs, California, will close its doors on 26 April, shutting down a unique telescope that is just beginning to explore the low-frequency radio sky. A final effort to obtain funds from the National Science Foundation (NSF) fell through at the end of March.

CLRO operates in the range of $15-125$ $\mathrm{MHz}$, "unfashionably" low for much of radioastronomy, but with a dedicated group of enthusiastic users. CLRO played a critical part in the discovery of the millisecond pulsar; the characteristically steep spectra of such pulsars are conveniently detectable at low frequencies. The phased array at CLRO is also much more sensitive than other low-frequency instruments. CLRO recently completed a catalogue of low-frequency radio sources, increasing their number to nearly 35,000 from the 350 previously identified.

CLRO also provides the only twodimensional imaging of the solar corona, according to Mukul Kundu, a professor of astronomy at the University of Maryland and one of the observatory's directors. The telescope can yield images of emission patterns at a rate of one every 10 milliseconds, and so can allow observation of rapid phenomena in the corona.

The University of Maryland owns and operates CLRO but operating funds have come primarily from NSF. When NSF "pulled the plug", the university was loth to provide the minimum $\$ 200,000$ per year needed to keep the facility going; it did not help that the observatory was 3,000 miles away.

With a lot of prodding, the university agreed to provide just under half that sum if NSF would make up the difference. But when NSF declined at the end of March, Maryland gave notice to the four full-time employees at CLRO.

Bad timing has played a part in CLRO's demise. Three years ago, when NSF was evalulating CLRO's status, the telescope was still being calibrated. Only after NSF took the decision to scale down support did exciting results begin to emerge. Moreover, because of its small size, CLRO lacked the political muscle to rally the astronomical community to its aid.

At least a two-month reprieve may come from the Office of Naval Research. Erickson has interested the US Navy in using CLRO to investigate ionospheric phenomena.

Spurred by this initial interest, Erickson is planning to "knock on doors in Washington" to sell to others the virtues of CLRO as a tool for ionosphere research. But without a long-term grant, CLRO faces a hand-to-mouth existence for the foreseeable future. Joseph Palca 